\title{
DEVELOPMENT, REPAIR AND REGENERATION OF THE RETINAL PIGMENT EPITHELIUM
}

\author{
IAN GRIERSON ${ }^{1}$, PAUL HISCOTT $^{1}$, PENNY HOGG ${ }^{1}$, HELEN ROBEY $^{2}$, ANK MAZURE $^{2}$ and \\ GENEVIEVE LARKIN ${ }^{2}$ \\ Liverpool and London
}

\begin{abstract}
SUMMARY
An overview is presented of the retinal pigment epithelium (RPE) cell in repair and regeneration. Changes in the RPE associated with repair activities have been described as metaplasia. However, evidence is presented to show that RPE cells do not become either fibroblasts or macrophages but merely adopt the appearance of these cell types in pathological conditions. The phenotypic alterations seem to be substrate-related. The fibroblast form predominates on two-dimensional substrates rich in fibronectin and in three-dimensional collagen matrices. The macrophage form seems to be associated with insubstantial or inadequate substrates such as the vitreous, photoreceptor debris and some cell surfaces. In altered circumstances the dedifferentiated RPE can rapidly revert to an epithelioid form. However, the regeneration of an effective RPE mosaic is more difficult and dependent on many factors including the size of the initial lesion, the condition of the basement area, the status of the neuroretina and the existing pathology in the eye. The importance for the regeneration of a normal functioning RPE of the cells being out of the cell cycle, establishing effective junctioning, reorganising their cytoskeleton and having the required adhesive balance with the basement membrane is emphasised.
\end{abstract}

\section{NORMAL FEATURES AND DEVELOPMENT}

The retinal pigment epithelium (RPE) has a highly specialised organisation related to its functional role as an intermediary between the choroid and the neural retina. The RPE forms a mosaic of polygonal cells that apically are intimately attached to the outer segment tips of the photoreceptors while their bases rest on a basement membrane above Bruch's membrane. RPE cells are polarised and are involved in diverse activities essential to retinal

From: 'Unit of Ophthalmology, Department of Medicine, University of Liverpool, Liverpool and ${ }^{2}$ Department of Clinical Science, Institute of Ophthalmology, London, UK.

Correspondence to: Professor Ian Grierson, Unit of Ophthalmology, Department of Medicine, PO Box 147, Liverpool L69 3BX, UK. homeostasis and visual function. They phagocytose spent rod and cone outer segments, metabolise vitamin A, transport metabolites, synthesise glycosaminoglycans and constitute an important part of the blood-retinal barrier. Essential to the barrier are zonulae occludentes or tight junctions which prevent leakage through the intercellular clefts. Intermediate junctions (zonulae adherentes) have an adhesive role and also assist in maintaining the polygonal shape of the RPE by helping organise the superficial web of actin microfilaments.

Crucial to the maintenance of the integrity of the RPE are the numerous gap junctions which abound in the intercellular cleft and serve as low-resistance pathways for the passage of ions and metabolites between cells. ${ }^{2}$ These conduits are the means by which cell-to-cell cross-talk is achieved and may well be responsible for the generation of the $\mathrm{C}$-wave in the electroretinogram. RPE cells have an extremely limited replicative ability in situ and the necessary signalling or passage of inhibitors by way of the gap junction system is a likely route.

It is said that RPE cells do not have desmosomes and lack the keratin intermediate filaments which decorate these junctions in stratified epithelia. Certainly some species such as chick do lack keratin intermediate filaments in the cytoplasm of their RPE; ${ }^{3}$ the intermediate filament present is vimentin. ${ }^{3}$ There are now over 20 subtypes of keratin identified by biochemical or immunological means. Moll et al. ${ }^{4}$ classified the various keratin (or cytokeratin) subtypes on the basis of their molecular weight and charge. High molecular weight, basic keratins were graded with a low number (K1, K2, K3 etc) and low molecular weight, acidic keratins have a high number (K18, K19). The RPE of most mammals, including humans, has some keratins ${ }^{5}$ but not the large basic keratins which characterise stratified epithelia such as skin (rich in K1) and cornea (distinguished by the presence of K3). Equally, elaborate desmosomal junctions are not present between RPE cells although simple maculae adherens are to be found in some species but not in the human. Plackoglobin,

Eye (1994) 8, 255-262 C 1994 Royal College of Ophthalmologists 
a desmosomal and adhering junction protein, is associated with the RPE of all species but more desmosomal-specific proteins such as desmoplankin and desmoglein are absent from most species except for the cow and frog. ${ }^{6}$

Hemidesmosomes are vital for the effective anchorage of many epithelial sheets to their basement membrane. The anchorage of the RPE monolayer does not seem to require junctions of this level of sophistication and none are evident among the basal infoldings. Instead there seem to be a series of focal adhesions and focal contacts with the underlying substrate which are not much different from those which form in vitro when RPE cells are settled on a culture dish. ${ }^{7}$ On the other hand, a complex mix of biologically adhesive substances inhabits the basement membrane and Bruch's membrane. It is now known that laminin, fibronectin, collagens of various types and heparin and chondroitin sulphate proteoglycan ${ }^{8}$ are in intimate contact with the base of the RPE. This cocktail of substances not only adheres the cells to it, in the generally accepted chemical and physical sense, but also has a signalling role. Signalling between the extracellular matrix and a cell's cytoskeleton of cytoplasmic filaments has a behavioural effect on the cell itself and in the present case helps to maintain polarisation and the status quo.

The precursors of the RPE cells are derived from the neuroepithelial cells of the neural plate. Although subjected to neuralation like adjacent tissue such as the neuroretina and optic stalk, they remain separate and distinct by being restricted to the outer leaf of the eye cup. Following pigment cell induction it is not entirely clear when cells which are to follow a pigment epithelial lineage become irreversibly committed to that lineage. ${ }^{9}$ Equally, potential determinant factors and their origins remain to be identified. It is certainly the case that removal of the neuroretinal anlage in many species provokes the neuroepithelium to transdifferentiate into neuroretinal tissue. The capacity to transdifferentiate is lost with age but persists far longer in some groups of animal than others. In mammals and birds the capacity is lost relatively rapidly during development, whereas in frogs the capacity remains until metamorphosis and some salamanders retain the capacity for RPE transdifferentiation into neuroretina throughout life. ${ }^{9}$ It has been shown that fibroblast growth factor, particularly basic fibroblast growth factor which is produced by RPE, stimulates neuroretinal regeneration in the chick embryo where the retina has been surgically removed. ${ }^{10}$ The regeneration is dose dependent, so that fibroblast growth factor is therefore a likely candidate for one of the retinal inducers or so-called determinant factors.

In addition to neuroretinal transdifferentiation, RPE can also, with appropriate stimuli, form iris or lens epithelium in some species of urodeles. The process of transdifferentiation has been called metaplasia but the process is not one of a cell changing into another type of cell. It is more a situation of a multipotential cell, capable of going down a number of avenues, being directed in one way or another. The cell can change direction before it becomes irreversibly locked onto a particular pathway. ${ }^{11,12}$
The eye undergoes about three doublings in diameter between the third week and the fifth month of development. During this period the RPE increases by both proliferation and expansion, the most marked expansion being the early change from multilayer to monolayer. ${ }^{12,13}$ From a level of high proliferation at the fourth fetal month, at a time when the eye as a whole is in a period of rapid growth, ${ }^{13}$ the division rate tails off to the sixth month, ${ }^{12,13}$ but the RPE cells continue to synthesise at a high rate. RNA synthesis, for example, extends well into the postnatal period, slowing down much later than in the neural tissue. ${ }^{14}$ As well as contributing in a major way to the production of Bruch's membrane, RPE cells have a pivotal role in the induction and modulation of the development of the scleral coat.

Following the end of high levels of proliferation among RPE cells, further increase in the area of the layer as the coats of the eye enlarge requires the enlargement of individual cells. The average number of RPE per unit area decreases in all regions between the fourth and ninth months of gestation. ${ }^{15}$ Increase in cell numbers still goes on rapidly in the neural retina and as the RPE need to spread out, there is little close adhesion between the two layers at this stage. Indeed, until intimate contact between neural and epithelial layers is established, photoreceptor outer segments do not develop, highlighting a vital trophic role for the differentiating RPE. ${ }^{16}$ The trophic requirement, surprisingly, is not RPE specific, because when embryonic neuroretina is transplanted into rat brains the photoreceptors develop outer segments. Here the role of the RPE is taken up, to some extent, by microglia. ${ }^{17}$

\section{REPAIR AND DEDIFFERENTIATION}

Under normal conditions the RPE cells are stationary and mitotically feeble and, as mentioned earlier, this state is established in development. Over the first few decades the area occupied by the RPE expands substantially from about $8 \mathrm{~cm}^{2}$ at 2 years to over $11 \mathrm{~cm}^{2}$ at 45 years, but RPE cell numbers remain at around 400000 for that period. ${ }^{18}$ As in development, much of the RPE expansion depends on the enlargement of individual cells. Enlargement is particularly marked in the periphery, where RPE cells can become binucleate or even multinucleate and measure upwards of $60 \mu \mathrm{m}$ in diameter compared with $14 \mu \mathrm{m}$ for the cells in the macula. ${ }^{19}$ The RPE cells have not compromised their proliferative potential; it is merely suppressed. ${ }^{9}$ RPE cells will proliferate quite happily in tissue culture conditions, they often undergo division following injury and RPE mitosis is associated with a number of ocular pathologies.

RPE differs from other epithelium in that the cells originate from the neural ectoderm. ${ }^{9}$ Perhaps as a result they have a dichotomy of activities, some being characteristically epithelial while others are not. Their strong polarity and transport functions are epithelial activities. On the other hand they are intimately associated with the neural retina and serve an accessory function somewhat akin to that of glia in the brain. As with glia, RPE cells have a 
prominent wound-healing role. They may undertake a more generalised phagocytic action than the very specialised and highly synchronised phagocytosis of spent discs which characterises their tertiary form; alternatively, RPE cells become involved in fibrosis. The two injury-related activities have distinctive phenotypes which are quite different from that of the regular sedentary RPE cell. One form has the morphology of a large macrophage and the other is elongated, bipolar and has the features of a fibroblast (Fig. 1).

Machemer and co-workers ${ }^{20.21}$ studied the RPE following complex retinal detachment and concluded from animal models that the RPE cell is the key player in scar formation on the surface of the retina and that RPE proliferation and migration lead to the formation of epiretinal membranes (sheets of scar tissue). They identified the macrophage and fibroblast types of RPE cell following detachment and noted that they were actively proliferating and migratory, which of course are not normal features of the RPE. They were so impressed with the phenotypic change in the RPE that they described it as being metaplasia. ${ }^{20}$ Since then the concept of RPE metaplasia leading to membrane formation in conditions such as proliferative vitreoretinopathy has held sway. Supportive evidence has come from a number of subsequent investigations. Mandelcorn and co-workers ${ }^{22}$ showed that when cultured RPE cells were injected into the rabbit vitreous they metaplased into macrophage and fibroblast cells. That these cells arose from RPE and not from other cell types was confirmed when the 'metaplasia' still took place when cultured RPE cells were placed in a cell-tight diffusion chamber in the rabbit vitreous. ${ }^{23}$ Finally, when VidaurriLeal et $a l .^{24}$ took cultures of hexagonal to oval RPE cells and overlaid these with vitreous, the RPE cells invaded the three-dimensional matrix and became bipolar fibroblastlike cells.

The process should not be considered to be metaplasia because the RPE cells do not become macrophages and fibroblasts: they merely adopt their appearance. In addition, in altered circumstances RPE cells rapidly revert to an epithelioid phenotype. Some of our own studies help to illustrate the point. If cultured bovine or human RPE cells are taken from a culture dish at a time when they are reg-

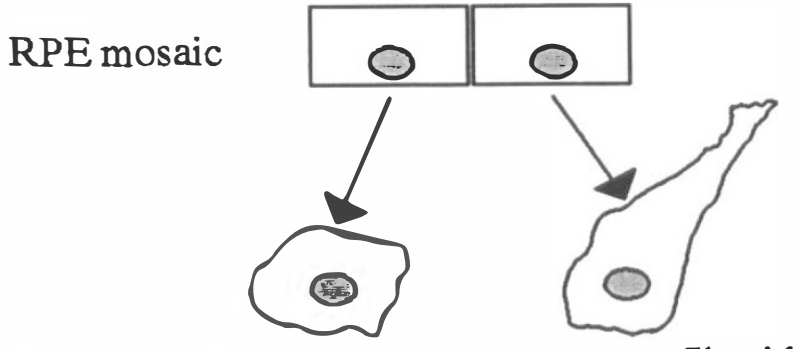

macrophage

fibroblast

Fig. 1. Following injury to the retinal pigment epithelium (RPE) mosaic, the RPE gives rise to two phenotypes of repair cell: a macrophage-like RPE cell and a fibroblast-like RPE cell. The phenotypic change is associated with the RPE becoming active, mobile and proliferative. (Derived from Machemer and Laqua. $^{20}$ ) ular, polarised and forming a mosaic and are introduced into the middle of a three-dimensional collagen matrix then they elongate into a bipolar, fibroblastic form. The fibroblast-like RPE cells migrate to the surface of the collagen and then spread out. ${ }^{25}$ Almost immediately they revert to a discoid shape and, with sufficient packing, form a loose mosaic. Transmission electron microscopy shows that in regions of high density where there is tight packing, gap junctions form between adjacent cells. During their passage through the collagen the RPE cells contract the matrix down until it is a small fraction of its former size. ${ }^{25}$ In addition they synthesise a wide range of extracellular materials such as the glycoprotein fibronectin on their passage through the framework.

Indeed if RPE cells are settled on a porous membrane in a migration chamber (Boyden chemoattraction chamber) and induced to migrate to a soluble chemoattractant placed in the well beneath the membrane, they go from epithelioid to fibroblastic when they start to move. The mobile RPE cells extend processes into the pores, stretch down and then pass through the pores to the other side of the membrane following the gradient of the attractant. ${ }^{26}$ Once on the under-surface of the membrane the RPE cells lose their fibroblastic shape and become flattened, epithelioid and stationary (Fig. 2). They do not form a mosaic, perhaps because the assay does not allow their numbers to become sufficiently large. Migration is associated with dramatic alterations in the RPE cytoskeleton. The pattern of actin staining goes from diffuse on the upper surface for settled cells, filamentous when they are spreading and migrating through pores (fibroblast phenotype), and finally to filaments organised into bundles called stress fibres when they are stationary on the undersurface of the membrane. ${ }^{26}$ Stress fibres are a feature of the normal RPE cell in situ and immobile, strongly adherent cells in vitro. ${ }^{27}$ Quite pronounced changes take place in the cytokeratins. RPE cells express a number of middle-range cytokeratins (e.g. K8) in situ but lack the large basic forms, and staining to detect the smallest acidic types (K18, K19) is inconclusive. ${ }^{5}$ Both $\mathrm{K} 18$ and $\mathrm{K} 19$ are expressed by cells migrating or in the process of migrating through pores (fibroblast phenotype) but not by the stationary epithlelioid cells (Fig. 3). It would therefore seem that the immunohistochemical demonstration of low molecular weight cytokeratins is an indicator of the fibroblastic migratory phenotype. ${ }^{26}$ To a large extent this is borne out by observations on pathological material, where $\mathrm{K} 18$ at least can be seen to stain only a subpopulation of the RPE cells that for the most part seem to be mobile. ${ }^{28}$

Characteristically both the macrophage and fibroblastic forms are mitotically active and migratory. The macrophage-like cell is a non-specific phagocyte which in pathological specimens and experimental in vivo injury models can be seen in the subretinal space and sometimes in the vitreous cavity. Undoubtedly the macrophage form inhabits the vitreal cavity in proliferative vitreoretinopathy as a component of the 'tobacco dust'. Tobacco dust is a colourful clinical description of vitreal aggregates of 

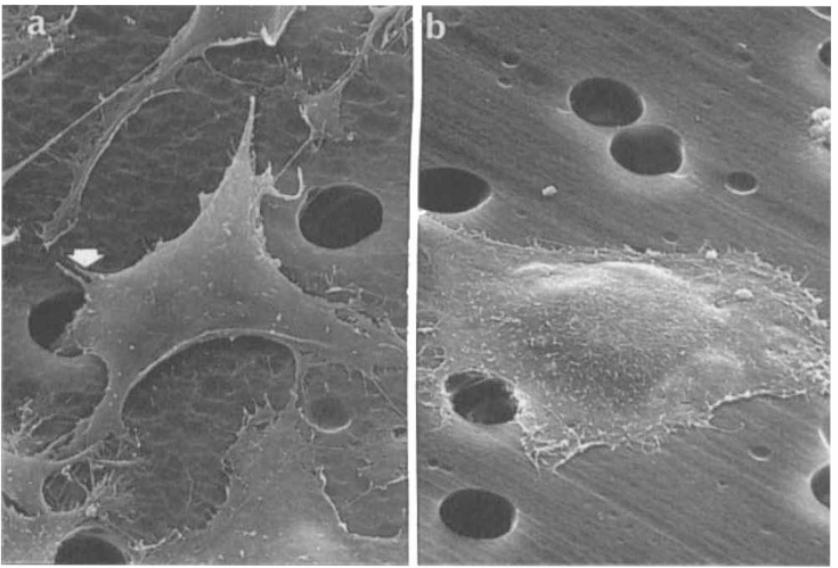

Fig. 2. Scanning electron micrographs of human RPE cells migrating in a microchemoattraction chamber to a stimulus of $10 \mu \mathrm{g} / \mathrm{ml}$ soluble bovine fibronectin. (a) The actively mobile $R P E$ on the upper surface of the perforated polycarbonate membrane have a fibroblastic morphology and one can be seen (arrow) dipping into a pore. $\times 1500$. (b) When the RPE have migrated through the pores, they become immobile and epithelioid. $\times 1200$.

mobile RPE (and possibly released melanin) which have passed through retinal holes and will eventually become part of the developing epiretinal membranes. As with the fibroblast terminology, calling the phagocytic RPE cells 'macrophages' is purely descriptive and functional and not generic. They remain RPE cells on the basis of cytokeratin staining ${ }^{28-30}$ and do not become true macrophages - at least as far as can be determined on the basis of markers for mononuclear phagocyte system (MPS) cells. $^{31}$

The macrophage phenotype develops as soon as RPE cells are placed in an aqueous environment or when they are on an institutional substrate such as photoreceptor debris in the subretinal space or loose collagen fibrils in the vitreous.$^{21,22,24}$ Substrate seems to be crucial in determining whether a macrophage or fibroblast phenotype is

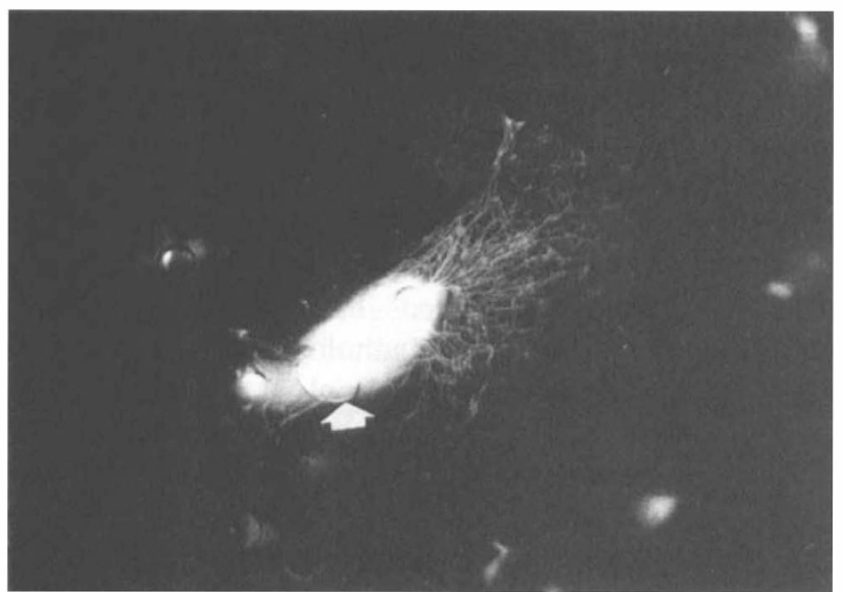

Fig. 3. Immunofluorescence photograph showing a cultured human RPE cell in the process of migrating through a pore (arrow) in the polycarbonate membrane of a microchemoattraction chamber. RPE with a fibroblast phenotype in the process of migrating stain positively with antibodies against the cytokeratin K18. $\times 400$. adopted. The fibroblast form is always seen if RPE cells are in a substantial three-dimensional collagen matrix, whether it be in a fibrous epiretinal membrane in vivo or a collagen gel in vitro. ${ }^{25}$ The fibroblast form develops on two-dimensional surfaces, particularly when cell density is low and the surface is rich in fibronectin. In the absence of fibronectin and under the pressure of crowding, an epithelioid phenotype is readily adopted.

\section{REGENERATION}

For the purposes of this paper, we have defined repair as the circumstances during which the dedifferentiated macrophage-like and fibroblast-like forms of the RPE cell hold sway. Regeneration, on the other hand, results in the establishment of a functionally intact monolayer of nondividing, tertiary differentiated cells (Fig. 4). From the previous section we can see that, although the RPE in injury is dramatically different in appearance and behaviour from the normal RPE, when conditions alter then the repair forms can rapidly revert to a more epithelioid form. Although taking up the epithelial form is not problematic, it seems to be far more difficult to get the RPE to reestablish an effective and functional mosaic both in vitro and in vivo.

When considering how the RPE could become regenerated it is worth bearing in mind what helps maintain the status quo normally and therefore what needs to be reconstituted. The RPE cells need to: (1) be out of the cell cycle, (2) downregulate their mitogen- and motogen-related receptor systems, (3) establish cell-to-cell communication, (4) synchronise their activities, (5) adjust their cytoskeleton so that they are polyglonal for optimum packing, (6) reinstate barrier conditions and (7) develop the required adhesion mechanisms for contact with the basement membrane basally and photoreceptors apically.

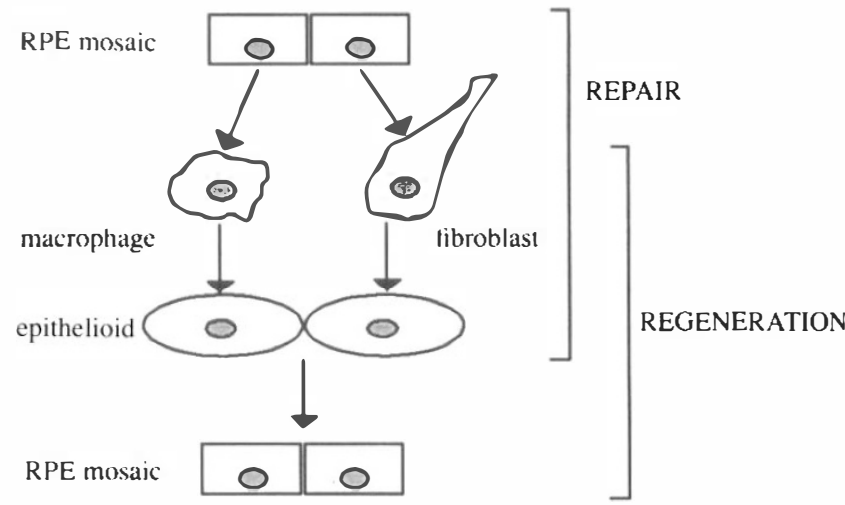

Fig. 4. For convenience, repair of the RPE is considered to involve the dedifferentiation of stationary non-dividing cells into migratory, proliferation macrophagic or fibroblastic forms. In turn the macrophagic and fibroblastic cells can become sedentary and exit the cell cycle and these we call epithelioid. The repair stage ends with the formation of epithelioid cells which form a sheet but not a proper mosaic. Regeneration covers the stages from the dedifferentiated repair cells, to epithelioid cells and through to mosaic formation. The cells of the RPE mosaic not only are sedentary and non-dividing like epithelioid cells but, in addition, have re-established barrier conditions and are polarised. Formation of a mosaic seems to be far more difficult than any other step. 
Maintenance of tertiary structure and polarisation depends on numerous signals. For example, undergoing synchronised activities such as transport of metabolites and phagocytosis of outer segments are reinforcing stimuli. The RPE stores and metabolises large amounts of vitamin A. Retinoids have growth-inhibiting properties and also can be differentiating agents. It is now known that the cytoskeleton in mesothelial cells is influenced by vitamin $\mathrm{A},{ }^{32}$ which seems to regulate intermediate filament expression in a number of simple epithelia. As the cytoskeleton is extremely important in the maintenance of cell shape and behaviour, retinoids may well have, in addition to their direct effect on proliferation, an indirect influence on tertiary structure via the cytoskeleton. Differentiating agents such as retinoids and butyrate seem to have a growth-inhibiting effect on ocular cells ${ }^{33.34}$ including $\mathrm{RPE},{ }^{35}$ at least as far as in vitro studies have been able to show.

Cell-to-cell communication via gap junctions is essential both to maintain synchrony and probably also to inhibit cell division. ${ }^{2,36}$ Evidence for gap junctional involvement in the control of RPE division is still lacking, but other ocular tissues where the cells have similar behavioural characteristics to RPE, such as trabecular meshwork cells, corneal endothelial cells and lens epithelial cells, exhibit strong contact inhibition of division and gap junctions abound in their intercellular clefts. Tight junctions and intermediate junctions provide the effective cellto-cell adhesion mechanisms for RPE. It may well be that a combination of the zonulae adherents and the cytoplasmic web of actin microfilaments establish efficient cell-tocell packing in the typical polygonal form, although how this packing is achieved remains obscure. ${ }^{37}$

Strong adhesion to the basement membrane is crucial not only to inhibit migration but also to influence a wide range of behavioural activities. It is becoming increasingly clear that the extracellular matrix imparts structural and functional signals to the cells which rest on it and the process is sometimes called dynamic reciprocity. The key players are the components of the extracellular matrix, receptors and adhesional modifications necessary to link matrix components to the cell plasma membrane and the cytoskeleton of the cell responsible for cell shape. ${ }^{38}$ It is now known from studies of epidermal repair that fibronectin is replete in injury while the cells are migrating and filling the lesion (Fig. 5). However, when the keratinocytes have filled in the lesion and started to differentiate, laminin becomes the dominant glycoprotein and fibronectin is in short supply. It seems that one glycoprotein is required in injury but the other is needed for terminal differentiation.

Receptors which link cells to the extracellular matrix include those of the integrin family and receptors of this type have been located on RPE. ${ }^{39.40}$ Once in the cell cycle, when the RPE cells lose their strong adhesion to the basement membrane, it has not yet been shown but could be predicted that there are alterations to the integrin receptor profile. A denser concentration of adhesion receptors is associated with focal contacts. These adhering contacts are rich in vinculin and are associated with actin microfilaments from the cytoskeleton. ${ }^{41}$ Obviously the correct integrin density and distribution of focal contact-like adhesions needs to be created if effective regeneration is to be achieved. Before cuboidal epithelial cells migrate and proliferate, they change shape and usually flatten and spread. It is now known that adhesion-related shape change, by dramatically altering the cytoskeleton, provokes entry into the cell cycle and activation of the genes associated with the $\mathrm{G}_{0}$ and $\mathrm{G}_{1}$ transition, particularly c-fos and c-myc. ${ }^{42}$ Extracellular matrix and cytoskeletal interactions which alter cell shape may regulate the transduction of mitogenic signals to the nucleus. ${ }^{43,44}$

Thus a host of controlling mechanisms serve to maintain the RPE mosaic in its non-proliferating, non-migratory, polarised and synchronised form against stimuli released after injury or as pathology develops which would lead to dedifferentiation and adoption of the injury phenotypes. It has to be borne in mind that the controls have all to be re-established if a competent mosaic of polarised RPE is to be regenerated. It is important to
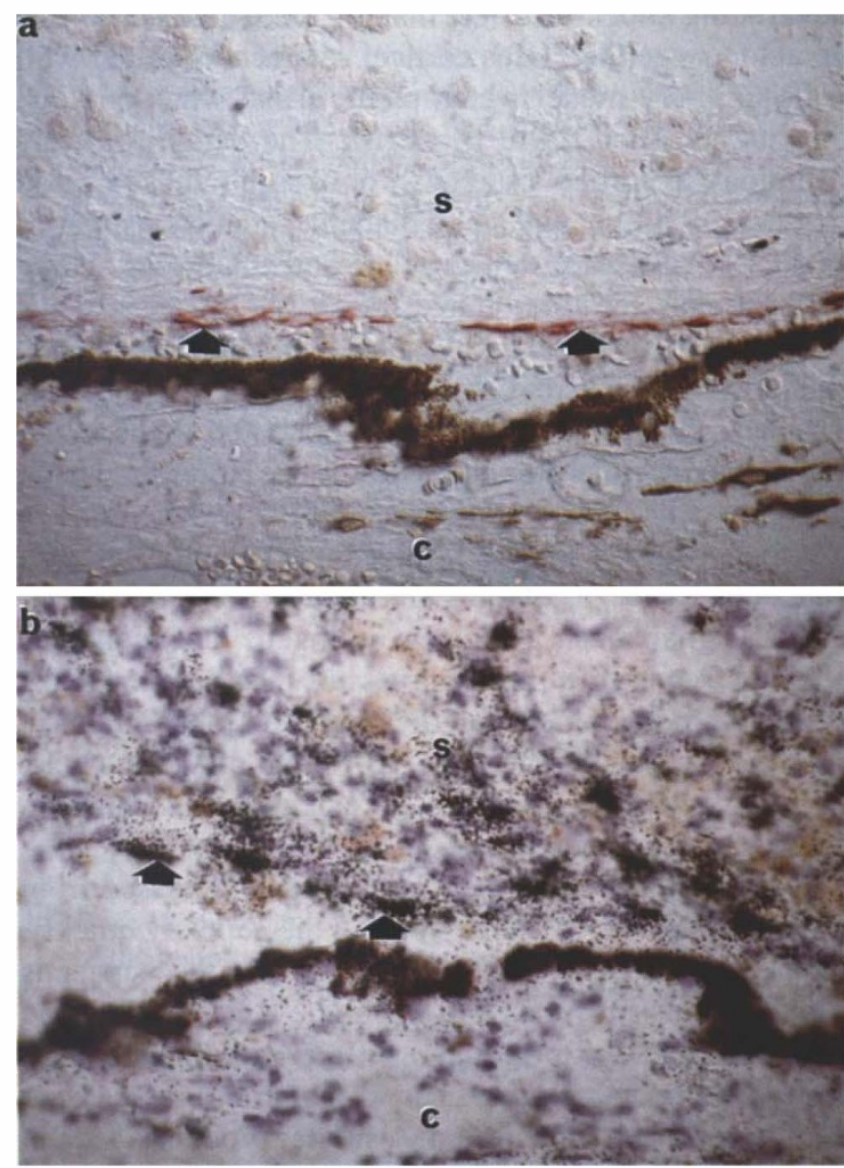

Fig. 5. Sections from a subretinal proliferation $(s)$ with subjacent retinal pigment epithelium and choroid (c). (a) Stained with the immunoperoxidase method (no counterstain) for cytokeratin 18 (CK18). A prominent layer of CK18-positive $R P E$ cells (arrow's) is present in the subretinal proliferation. (b) Labelled with an anti-sense probe for fibronectin $m R N A$ employing an autoradiographic in situ hybridisation technique. Many of the migratory RPE cells show intense labelling for fibronectin $m R N A$ (arrow's). $\times 400$. 
appreciate what happens when the mosaic is stressed and synchrony breaks down in a strongly contact-inhibited system such as the RPE. A model of breakdown of synchrony and contact inhibition is shown in Fig. 6. The model assumes that, as with other synchronised systems, cell-to-cell communication can be closed down to protect cells adjacent to a lesion.

It has been hypothesised that the low-resistance pathways via the connexons of gap junctions become highresistance pathways in injury to avoid sodium pump failure and a domino effect. ${ }^{45}$ The process at the gap junction level has been described as crystallisation ${ }^{45}$ and a key switch would seem to be intracellular calcium levels. Crystallisation is a necessary defence mechanism somewhat analogous to sealing off watertight doors on a ship when one compartment has been holed. An idea of how necessary a switch-off mechanism is can be gleaned from close investigation of the lens. Lens fibres are an extremely rich source of gap junctions but these junctions seem not to be able to crystallise. ${ }^{45}$ Why the switch-off mechanism is inoperative in lens fibre cells is unknown. However, it does mean that sporadic cell death can have a devastating knock-on effect making the whole tissue vulnerable to swelling. Lens cortical cataracts fit rather well with an injury model which predicts that when one cell dies its neighbours will be at risk. The pattern of early pathology in cortical cataracts, for example those caused by ultraviolet light and some diabetic cataracts, is that of small focal densifications growing and coalescing. Many suffer because of a lesion in a few.

Most epithelial sheets have a calcium, and perhaps $\mathrm{pH}$, shutdown mechanism. Let us consider a focal lesion here. The cells adjacent to the lesion are isolated from harm but are isolated from each other as well: the larger the lesion the more cells in isolation (Fig. 6). Clearly synchrony is lost to a greater or lesser extent and the cells for the most part are free to act as isolated units and respond to environmental signals such as soluble mitogens and motogens released from the dead or dying tissue. In addition, contact inhibition of movement and division is no longer in operation so eventually the cells will enter the cell cycle. Growth factor receptors are upregulated and adhesion mechanisms downregulated.

If the lesion is small then adjacent cells released from contact inhibition will slide in to fill the gap very quickly. Tight junctions will be re-formed so that the barrier is complete, gap junctions will open, the RPE will become polarised, and the status quo will be established once more. All that will remain is an irregularity in cell size at the site of the erstwhile lesion. A similar process is seen in the corneal endothelium where filling of defects can be followed by specular microscopy. Previous deficits are covered either by giant cells (where one or two cells have moved in to fill the gap) or by florets (where several cells simultaneously have moved in to fill the larger gap). In the RPE, such events are associated with mild photocoagulation lesions and limited retinal detachment.

Problems start to arise when the focal lesion is large or a
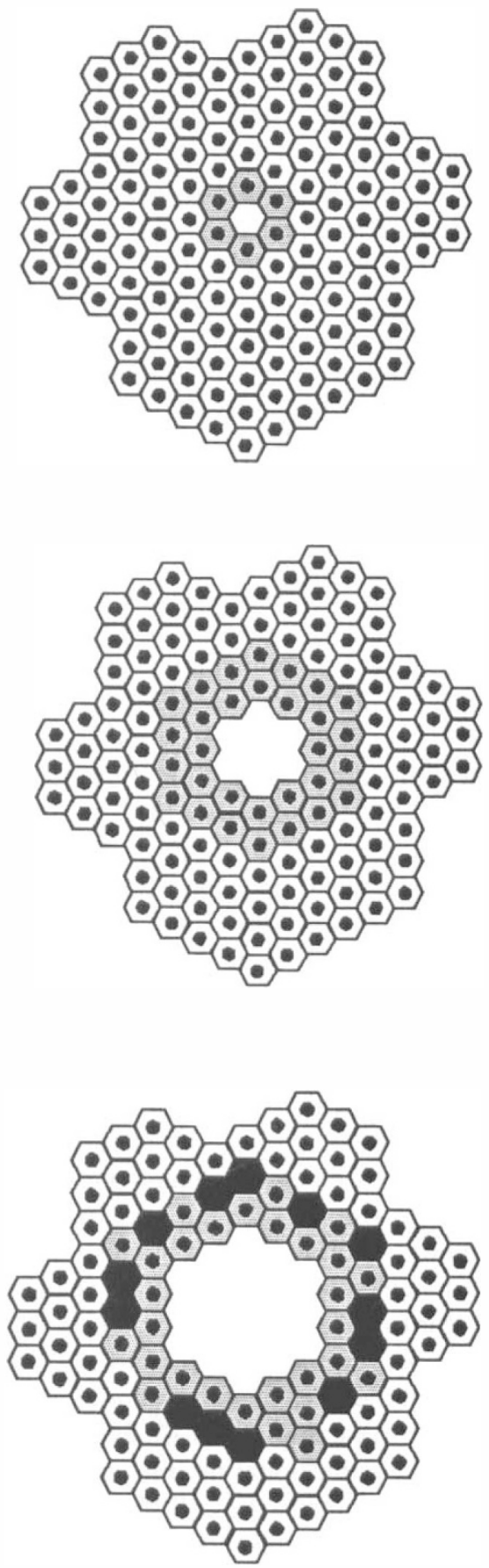

Fig. 6. Drawings of part of the RPE mosaic, where white cells are contact-inhibited, grey cells have lost contact inhibition and black cells are mitotic. (a) The clear area in the centre of the mosaic is a dead cell, adjacent cells have isolated themselves by making the pathways via gap junctions of high resistance. The deficit is repaired by enlargement and sliding in of the isolated cells. (b) Larger deficits can be filled by the same mechanism but more cells are isolated and the refill time is longer. Once the deficit is filled, contact inhibition is re-established. (c) At some point the deficit becomes so large that environmental stimuli overcome the inhibition of division and some cells enter the division cycle. The deficit is filled in by a combination of migration and proliferation. Contact inhibition can become re-established but in a number of circumstances (e.g. if the basement layer is damaged, if the lesion is particularly large or mitogens are present in high concentrations for protracted periods) a monolayer is not re-established and excessive proliferation takes place. 
the lesion is diffuse. In these circumstances the situation may be complicated by deficits in Bruch's membrane (destroying the template for rapid regeneration), there being widespread ocular pathology (filling the surrounding media with cytokines) or retinal detachment (depletion of differentiation agents such as vitamin $\mathrm{A}$ and release of cytokines). Junctional integrity is interrupted for so long that the injury phenotypes (macrophage-like and fibroblast-like) have the opportunity to become fully established, growth factor receptors are upregulated in a massive way, synthesis of injury-related glycoproteins such as fibronectin takes place and the cells actively migrate and proliferate in a non-coordinated manner.

Whether or not an effective RPE monolayer is regenerated depends to a large extent on how disrupted the architecture has become with respect to not only the RPE and Bruch's membrane but also the neural retina. The repair and regenerative process can be seen to good effect not only following long-standing retinal detachment ${ }^{20.21}$ but also, for example, after laser burns,${ }^{46}$ intense light exposure $^{47}$ and penetrating trauma. ${ }^{48}$ RPE repair ending in a regenerated monolayer (regenerative repair) is the province of the small lesion. ${ }^{18}$ In large diffuse lesions the characteristic feature is for overexuberant proliferation and migration of RPE cells which does not become selflimiting until advanced changes have taken place. Mounds of RPE can develop on Bruch's membrane and these can lead to the formation of extended multilayers of spindleshaped RPE, as can happen for example after long-standing detachment and if there is malignant melanoma of the choroid. ${ }^{49}$ Subretinal and epiretinal involvement of RPE in membranes is the inevitable consequence. Subretinal columnar and tubular arrangements of RPE are sometimes formed, while at the ora serrata RPE proliferation is involved in ringschwiele formation. Migration of RPE into the retina is common in end-stage detachments and retinal degenerations. In retinitis pigmentosa the migrating RPE cells become associated with retinal vessels forming the so-called bone spicule pattern. ${ }^{50}$

An important recent development is experimental RPE transplantation. Tissue cultured normal RPE cells have been injected onto Bruch's membrane of RCS rats as a means of halting or delaying the hereditary degeneration which emerges in this animal model. The photoreceptor degeneration which develops is known to be secondary to a lesion developing in the RPE cells, so replacing them with normal cells is a logical means of trying to preserve vision. The results have been sufficiently encouraging to raise hopes (perhaps premature) that transplantation may be a way of treating retinal diseases in which the RPE has an important role. ${ }^{51}$

There are many, many difficulties with RPE transplantation before it can be of value to human retinal disease and a number of major hurdles need to be overcome. Appropriate microsurgical technology is required. Rejection problems have to be addressed. However, unless the control of tertiary differentiation of RPE cells is understood, injection of proliferating RPE onto Bruch's mem- brane may not only be pointless, it may even be dangerous. Work needs to be done in exploring the factors and conditions which are required to establish cell-to-cell contact inhibition of division and a fully differentiated RPE mosaic.

Support is provided by the Littlewoods Foundation and the Foundation for the Prevention of Blindness. Our research has been funded by the T. F. C. Frost Foundation, Fight for Sight and the Guide Dogs for the Blind Association. I.G. is a Visiting Senior Fellow to the Department of Clinical Science, Institute of Ophthalmology, London.

\section{REFERENCES}

1. Sandig M, Kalnins VI. Subunits in zonulae adhaerentes and striations in the associated circumferential microfilament bundles in chicken retinal pigment epithelial cells in situ. Exp Cell Res 1988:175:1-14.

2. Hudspeth AJ, Yee AG. The intercellular junctional complexes of retinal pigment epithelia. Invest Ophthalmol 1973; 12:354-65.

3. Docherty RJ, Edwards JG, Garrod DR, Mattey DI. Chick embryonic pigmented retina is one of the group of epithelioid tissues that lack cytokeratins and desmosomes and have intermediate filaments composed of vimentin. J Cell Sci 1984;71:61-74.

4. Moll R, Franke WW, Schilier DL. The catalog of human cytokeratins: patterns of expression in normal epithelia, tumours and cultured cells. Cell 1982;31:11-24.

5. McKechnie NM, Boulton M, Robey HL, Savage FJ, Grierson I. The cytoskeletal elements of human retinal pigment epithelium: in vitro and in vivo. J Cell Sci 1988;91:303-12.

6. Owaribe K, Kartenbeck J, Rungger-Brandle E, Franke WW. Cytoskeletons of retinal pigment epithelial cells: interspecies differences of expression patterns indicate independence of cell function from the specific complement of cytoskeletal proteins. Cell Tissue Res 1988;254:301-15.

7. Opas M, Kalnins VI. Light-microscopical analysis of focal adhesions of retinal pigmented epithelial cells. Invest Ophthalmol Vis Sci 1986;27:1622-33.

8. Lin W-L, Essner E, McCarthy KJ, Couchman JR. Ultrastructural immunocytochemical localization of chondroitin sulfate proteoglycan in Bruch's membrane of the rat. Invest Ophthalmol Vis Sci 1992;33:2072-5.

9. Coulombre AJ. Roles of the retinal pigment epithelium in the development of ocular tissues. In: Zinn KM, Marmor MF, editors. The retinal pigment epithelium. Cambridge, Mass.: Harvard University Press, 1979.

10. Park CM, Hollenberg MJ. Basic fibroblast growth factor induces retinal regeneration in vivo. Dev Biol 1989;134:201-5.

11. Buse E, Eichmann T, de Groot H, Leker A. Differentiation of the mammalian retinal pigment epithelium in vitro: influence of presumptive retinal neuroepithelium and head mesenchyme. Anat Embryol 1993;187:259-68.

12. Stroeva OG, Mitashov VI. Retinal pigment epithelium: proliferation and differentiation during development and regeneration. Int Rev Cytol 1983;83:221-93.

13. Mann I. The development of the human eye. London: British Medical Association, 1949.

14. Kong Y, Usuda N, Morita, T, Hanai T, Nagata T. Study on RNA synthesis in the retina and retinal pigment epithelium of mice by light microscope autoradiography. Cell Mol Biol 1992;38:669-78.

15. Streeten BW. Development of the human retinal pigment epithelium and the posterior segment. Arch Ophthalmol 1969;81:383-94.

16. Hollifield J, Witkovsky P. Pigmented retinal epithelium 
involvement in photoreceptor development and function. Exp Zool 1974;189:357-77.

17. Banerjee R, Lund RD. A role for microglia in the maintenance of photoreceptors in retinal transplants lacking pigment epithelium. J Neurocytol 1992;21:235-43.

18. Tso MOM. Developmental, reactive and neoplastic proliferation of the retinal pigment epithelium. In: Marmour MF, Zinn KM, editors. The retinal pigment epithelium, Cambridge Mass.: Harvard University Press, 1979:267-76.

19. Hogan MJ, Alvarado JA, Weddell JE. Histology of the human eye. Philadelphia, Saunders: 1971.

20. Machemer R, Laqua H. Pigment epithelial proliferation in retinal detachment (massive periretinal proliferation). Am J Ophthalmol 1975;80:1-23.

21. Machemer R, van Horn D, Aaberg TM. Pigment epithelial proliferation in human retinal detachment and massive periretinal proliferation. Am J Ophthalmol 1978;85:181-91.

22. Mandelcorn MS, Machemer R, Fineberg E, Hersch SB. Proliferation and metaplasia of intravitreal retinal pigment epithelial cell autotransplants. Am J Ophthalmol 1975;80:227-37.

23. Muller-Jensen K, Machemer R, Azarnia R. Autotransplantation of retinal pigment epithelial cells in intravitreal diffusion chamber. Am J Ophthalmol 1975;80:530-7.

24. Vidaurri-Leal J, Hohman R, Glaser BM. Effect of vitreous on morphologic characteristics of retinal pigment epithelial cells. Arch Ophthamol 1984;102:1220-3.

25. Mazure A, Grierson I. In vivo studies of the contractility of cell types involved in proliferative vitreoretinopathy. Invest Ophthalmol Vis Sci 1992;33:3407-16.

26. Robey HL, Hiscott PS, Grierson I. Cytokeratins and retinal epithelial cell behaviour. J Cell Sci 1992;102:329-40.

27. Badley RA, Couchman JR, Rees DA. Comparison of the cell cytoskeleton in migratory and stationary chick fibroblasts. J Muscle Res Cell Motil 1980;1:5-14.

28. Hiscott P, Morino I, McKechnie N, Grierson I, Gregor Z. Proliferating retinal pigment epithelial cells in subretinal membranes. In: Khoo CY, Ang BC, Cheah WM, Chew PTK, Lim ASM, editors. New frontiers in ophthalmology. Amsterdam: Excerpta Medica, 1991: 1115-20.

29. Hiscott PS, Grierson I, McLeod D. Retinal pigment epithelial cells in epiretinal membranes: an immunohistochemical study. Br J Ophthalmol 1984;68:708-15.

30. Grierson I, Boulton M, Hiscott P, Hitchins C. Gilbert D, McLeod D. Human retinal pigment epithelial cells in the vitreous of the owl monkey. Exp Eye Res 1986;43:491-502.

31. Charteris DG, Hiscott P, Grierson I, Lightman SL. Proliferative vitreoretinopathy: lymphocytes in epiretinal membranes. Ophthalmology 1992;99:1364-7.

32. Kim KH, Stellmach V, Javors J, Fuchs E. Regulation of human mesothelial cell differentiation: opposing roles of retinoids and epidermoid growth factor in the expression of intermediate filament proteins. J Cell Biol 1987;105: 3039-51.

33. Khaw PT, Ward S, Porter A, Grierson I, Hitchings RA, Rice NSC. Long-term effects of 5-fluorouracil and sodium butyrate on human Tenon's fibroblasts. Invest Ophthalmol Vis Sci 1992;33:2043-52.
34. Kim RY, Stern WH. Retinoids and butyrate modulate fibroblast growth and contraction of collagen matrices. Invest Ophthalmol Vis Sci 1992;31:1183-6.

35. Camapochiaro PA, Hackett SF, Conway BP. Retinoic acid promotes density dependent growth arrest in human retinal pigment epithelial cells. Invest Ophthalmol Vis Sci 1991;32:65-72.

36. Warner A. The gap junction. J Cell Sci 1988;89:1-7.

37. Crawford BJ. Development of the junctional complex during differentiation of chick pigmented epithelial cells in clonal culture. Invest Ophthalmol Vis Sci 1980;19:223-37.

38. Stein WD, Bronner F. Cell shape: determinants, regulation, and regulatory role. San Diego: Academic Press, 1989.

39. Anderson DH, Guerin CJ, Matsumoto B, Pfeffer BA. Identification and localisation of a beta-1 receptor from the integrin family in mammalian retinal pigment epithelial cells. Invest Ophthalmol Vis Sci 1990;31:81-93.

40. Chu P, Grunwald GB. Functional inhibition of retinal pigment epithelial cell-substrate adhesion with a monoclonal antibody against the beta-one subunit of integrin. Invest Ophthalmol Vis Sci 1991;32:1763-9.

41. Opas M, Kalnins VI. Light-microscopical analysis of focal adhesions of retinal pigmented epithelial cells. Invest Ophthalmol Vis Sci 1986;27:1622-33.

42. Dike LE, Farmer SR. Cell adhesion induces the expression of growth associated genes in suspension-arrested fibroblasts. Proc Natl Acad Sci USA 1988;85:6792-6.

43. Burridge K. Substrate adhesions in normal and transformed fibroblasts: organisation and regulation of cytoskeletal, membrane and extracellular matrix components as focal contacts. Cancer Rev 1986;4:18-78.

44. Farmer SR, Ben-Ze'ev A, Benecke BJ, Penman S. Altered translatability of messenger RNA from suspended anchorage-dependent fibroblasts: reversal upon cell attachment to a surface. Cell 1978;15:627-37.

45. Goodenough DA. Lens gap junctions: a structural hypothesis for nonregulated low-resistance intercellular pathways. Invest Ophthalmol Vis Sci 1979;18:1104-22.

46. L'Esperance FA. The ocular histopathalogic effect of krypton and argon laser radiation. Am J Ophthalmol 1969;68:263-73.

47. T'so MOM. Photic maculopathy in the rhesus monkey: a light and electron microscopic study. Invest Ophthalmol Vis Sci $1973 ; 12: 17-34$

48. Cleary PE, Ryan SJ. Experimental posterior penetrating eye injury in the rabbit. II. Histology of wound, vitreous and retina. Br J Ophthalmol 1979;63:312-21.

49. Wallow IHL, T'so MOM. Proliferation of the retinal pigment epithelium over malignant choroidal tumours: a light and electron microscopic study. Am J Ophthalmol 1972;73:914-26.

50. Gardner S, Hendkind P. Pathology of retinitis pigmentosa. Ophthalmology 1982;89:1425-32.

51. Gouras P, Lopez R. Transplantation of retinal epithelial cells. Invest Ophthalmol Vis Sci 1989;30:1681-3. 